\title{
Focus on organic electronic devices
}

This special issue is devoted to the 4th Conference DIELOR 2010 (Organic Electronic Devices) which was held on October 11-13, at the peninsula of Giens in the south of France. DIELOR 2010 was aimed at addressing the more recent developments in the area of organic electronic devices, from materials to applications. Covered thematics during the conference include organic materials, organic solar cells, organic transistors, OLEDs, sensors and processing. The papers published in this special issue were selected by the DIELOR 2010 scientific committee and were reviewed according to the guidelines of the journal. The guest editors would like to thank the reviewers for their valuable support in reviewing the papers. We hope this special issue will contribute to the promotion of relevant research results in the exciting area of organic electronics.

J.J. Simon

IM2NP (Institut Materiaux Microélectronique Nanosciences de Provence) UMR CNRS 6242 - Domaine Universitaire de Saint-Jérôme, 13397 Marseille Cedex 20, France 